\title{
Multi-scale imaging of protein oxidation in mayonnaise
}

\author{
Suyeon Yang ${ }^{\mathrm{a}, 1}$, Machi Takeuchi ${ }^{\mathrm{b}, \mathrm{c}, 1}$, Heiner Friedrich ${ }^{\mathrm{b}, \mathrm{c}, \mathrm{d}, *}$, John P.M. van Duynhoven ${ }^{\mathrm{a}, \mathrm{e}, *}$, and Johannes Hohlbein ${ }^{\mathrm{a}, \mathrm{f}, *}$ \\ ${ }^{a}$ Laboratory of Biophysics, Wageningen University and Research, Stippeneng 4, 6708 WE Wageningen, the Netherlands; ${ }^{\text {b }}$ Laboratory of Physical Chemistry, Department of \\ Chemical Engineering and Chemistry, Eindhoven University of Technology, P.O. Box 513, 5600 MB Eindhoven, the Netherlands; ${ }^{\mathrm{C}}$ Institute for Complex Molecular Systems, \\ Eindhoven University of Technology, P.O. Box 513, $5600 \mathrm{MB}$ Eindhoven, the Netherlands; ${ }^{\mathrm{d}}$ Center for Multiscale Electron Microscopy, Department of Chemical Engineering and

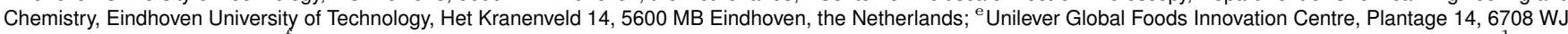 \\ Wageningen, the Netherlands; ${ }^{\mathrm{P}}$ Microspectroscopy Research Facility, Wageningen University and Research, Stippeneng 4, 6708 WE, Wageningen, the Netherlands; ${ }^{1}$ These \\ authors contributed equally to this work.; " Correspondence: john.vanduynhoven@wur.nl (J.v.D); H.Friedrich@tue.nl (H.F.); johannes.hohlbein@wur.nl (J.H.)
}

egg yolk protein oxidation | lipid oxidation | low-density lipoprotein particles (LDLs) | autofluorescence | cryo-TEM | protein free radical trapping light microscopy and cryogenic transmission electron microscopy. We further probed the formation of protein radicals and protein oxidation by imaging the accumulation of a water-soluble fluorescent spintrap and protein autofluorescence. The effect of variation of $\mathrm{pH}$ and addition of EDTA on accumulation of spintraps validated that protein radicals were induced by lipid radicals. We observed protein radical formation at both the oil/water droplet interface and in the continuous phase. Our data suggests two main pathways of oxidative protein radical formation in LDL particles: at the droplet interface, induced by lipid free radicals formed in oil droplets, and in the continuous phase induced by an independent LDL-specific mechanism.

In mayonnaise, lipid and protein oxidation are closely related and the interplay between them is critical for understanding the chemical shelf-life stability of mayonnaise. This is in particular the case for comprehending the role of low-density lipoprotein (LDL) particles as a main emulsifier. Here, we monitored oxidation and the concomitant aggregation of LDLs by bright field

\section{Introduction}

Mayonnaise derives its structural stability from egg yolk containing protein and lipid emulsifiers that reside at the oil/water droplet interface (Anton, 2013). The use of egg yolk as an emulsifier however also introduces strong pro-oxidants. Preventing oxidation of the oil phase and the proteins in mayonnaise has been a long-standing challenge, as oxidation can lead to reaction products that decrease physical stability, nutritional and sensorial value thereby limiting shelf-life (Berton-Carabin et al., 2014). Mayonnaise is formulated with vegetable oil, vinegar and egg yolk which contains phosvitin and low-density lipoprotein (LDL) particles that have a diameter of $40 \pm 20$ nm (Anton et al., 2003; Anton, 2013). LDL particles feature a core-shell structure where a core of triglycerides cholesteryl esters is surrounded by a monolayer of phospholipids and apolipoproteins. During the emulsification of mayonnaise, LDL particles can release phospholipids and apolipoproteins that together with phosvitin adsorb at the oil/water droplet interface. LDLs can also remain structurally intact and form granules present in the continuous phase or adsorbed at the oil/water interface depending on mechanical treatments and conditions (Anton et al., 2003; Mizutani and Nakamura, 1985; Sirvente et al., 2007).

Lipid oxidation in mayonnaise is mediated by free radical chain reactions (Schaich, 2012) and catalysed by the presence of metal ions such as iron introduced via phosvitin (BertonCarabin et al., 2014). Reactions between alkyl radicals (L·) and molecular oxygen generate peroxyl radicals (LOO·), which further accelerate lipid peroxidation by reacting with unsaturated lipids. Whereas at neutral $\mathrm{pH}$ pro-oxidant iron ions are strongly bound to the negatively charged phosvitin, a weaker binding at the acidic $\mathrm{pH}$ of mayonnaise makes them available as catalysts for lipid oxidation (Merkx et al., 2019). Therefore, ethylenediaminetetraacetic acid (EDTA) is often added to mayonnaise to bind free metal ions via chelation and thus reduces the formation of lipid peroxidation (LPO) products (Lee and Decker, 2011; Nielsen et al., 2004). In food emulsions, protein emulsifiers are known to react with free radicals formed via lipid oxidation thus acting as antioxidants via scavenging free radicals (Berton-Carabin et al., 2014; Schaich, 2008; Schaich and Karel, 1976; Wang et al., 2020; Berton et al., 2012). Emulsions stabilized by whey proteins, for example, showed better oxidation stability than emulsions stabilised by the surfactant Tween (Zhu et al., 2018). It has therefore been argued that due to their proximity to lipid radicals generated in the droplet phase, proteins adsorbed at the oil/water interface can function as effective antioxidants.

In mayonnaise, proteins that originate from LDL particles are either present at the oil/water droplet interface, as individual particles in the continuous phase, or aggregated in granules (Anton, 2013). Apoproteins from LDL particles can specifically act as emulsifiers at droplet interfaces within may- 
onnaise. In both intact LDLs and in dispersed form at droplet interfaces apoproteins can engage in oxidation reactions. The potential role of these particles in protein and lipid oxidation processes occurring in mayonnaise has been largely overlooked. Human plasma model systems have been used to study oxidation and aggregation of LDLs using bright field microscopy and transmission electron microscopy (TEM) (Xu and Lin, 2001). By means of UV/VIS and fluorescence spectroscopy, protein oxidation of LDL was investigated showing that the fluorescence intensity increased in the $430 \mathrm{~nm}$ emission range after auto-oxidation (Koller et al., 1986; Pinchuk and Lichtenberg, 1999, 2002). We hypothesize that the proteins from LDL particles can scavenge lipid radicals, not only in intact particles, but also in dispersed form at the droplet interfaces.

Here, we study oxidation in mayonnaise focusing on the structural aspects such as LDL aggregation and on localisation of protein free radicals at the oil/water droplet interface and in the continuous phase. Cryo-transmission electron microscopy (cryo-TEM) was used to observe LDL particles at the nanometre scale and we used plunge vitrification of LDLs present in the continuous phase isolated from mayonnaise to study oxidation in a time resolved manner. We further studied the impact of oxidation using optical bright field light microscopy which provides micro scale information allowing to identify aggregation of LDLs upon oxidation. Similar to biomedical approaches allowing to localise free radicals in vivo via fluorescent spin trapping adducts (Mason, 2016; Towner et al., 2012; Mason and Ganini, 2019), we developed a fluorescent spintrap CAMPO-AFDye 647 composed of the fluorophore AFDye 647 and CAMPO, a derivative of the water soluble spintrap DMPO. We monitored oxidation in dilute $(26 \%$ (w/w) oil concentration) mayonnaises and demonstrate the co-localisation of autofluorescence from oxidised proteins and CAMPO-AFDye 647 fluorescence from protein free radical spin adducts.

\section{Materials and Methods}

Materials. 2-((1E,3E)-5-((E)-3,3-dimethyl-5-sulfo-1-(3sulfopropyl)indolin-2-ylidene)penta-1,3-dien-1-yl)-3-methyl1-(6-((4-(2-methyl-1-oxido-3,4-dihydro-2H-pyrrole-2carboxamido)butyl)amino)-6-oxohexyl)-3-(4-sulfobutyl)3H-indol-1-ium-5-sulfonate (CAMPO-AFDye 647) and CAMPO-Au nanoparticles (CAMPO-AuNPs) were synthesized by SyMO-Chem B.V. (Eindhoven, the Netherlands). Reference AuNPs without CAMPO functionalization were obtained from AURION (Wageningen, the Netherlands). In the Appendix the molecular structures of these agents are shown (see Supplementary Fig. S1). Sodium chloride (>99.5\%, EMSURE®) and Rhodamine B were purchased from Sigma-Aldrich. Spirit white vinegar (4\%), soybean oil and egg yolk containing $8 \% \mathrm{NaCl}$ were purchased from a local store. Alumina powder (Alumina N-Super I) was obtained from MP EcoChrom ${ }^{\mathrm{TM}}$. EDTA (Ethylenediaminetetraacetic acid disodium salt dihydrate) was purchased from Merck. Demineralized (demi) water was used for all experiments.
Preparation of samples. Stripped soybean oil was prepared using alumina powder to remove lipid-soluble anti-oxidants (Berton et al., 2011). The oil was mixed with the powder at a volume ratio of 2:1 in Falcon tubes and shaken for $24 \mathrm{~h}$. The suspension was then centrifuged at $2000 \times g$ for $20 \mathrm{~min}$. The oil was collected, and the same centrifugation procedure was repeated to ensure complete removal of the alumina powder. Stripped soybean oil was used for the co-localization experiments of CAMPO-AFDye 647 and auto-fluorescence in dilute emulsions. Unless mentioned otherwise, non-stripped soybean oil was used.

Mayonnaise was prepared from $78 \%(\mathrm{w} / \mathrm{w})$ of soybean oil, $5 \%(\mathrm{w} / \mathrm{w}$ ) of egg yolk with $8 \% \mathrm{NaCl}, 0.7 \%$ (w/w) of salt, $14.8 \%$ $(\mathrm{w} / \mathrm{w})$ of demi water, and $1.5 \%(\mathrm{w} / \mathrm{w})$ of spirit vinegar using a Silverson mixer. Egg yolk, salt and demi water were premixed in a $500 \mathrm{~mL}$ jar and mixed at $2000 \mathrm{rpm}$ for $20 \mathrm{~s}$. Oil was slowly added and mixed at $8500 \mathrm{rpm}$ for 4 minutes. Spirit vinegar was added and mixed further for 2 minutes. For preparing a dilute mayonnaise, we added demi-water to reach a final $26 \%$ $(\mathrm{w} / \mathrm{w})$ oil concentration. Under standard conditions, the dilute mayonnaise had $\mathrm{pH}$ 4.0. Without addition of vinegar, the $\mathrm{pH}$ was 6.6. For samples formulated with EDTA, this ingredient was added to the water phase before making the emulsions.

The continuous phase of the prepared mayonnaise was obtained by centrifuging the fresh mayonnaise at $4000 \mathrm{~g}$ for 1 hour. The continuous phase was collected from the bottom part of the Eppendorf tubes by cutting off the bottom tip. The continuous phase was stored in a fridge at $4{ }^{\circ} \mathrm{C}$ until further use. To accelerate the oxidation, these separated continuous phases (each $100 \mu \mathrm{L}$ in $1.5 \mathrm{~mL}$ Eppendorf tubes) were stored at $40^{\circ} \mathrm{C}$ and sampled at $0,1,3$, and 5 days.

For oxidation experiments including spin traps, CAMPOAFDye $647(\mathrm{MW}=1068.30 \mathrm{~g} / \mathrm{mol})$ was dissolved with dimethylsulfoxide (DMSO) to prepare $0.01 \mathrm{~g} / \mathrm{L}$ of CAMPOAFDye 647 stock solutions. The stock solution was added to the continuous phase samples and mayonnaise to reach a final concentration of $1 \mathrm{\mu M}$. The CAMPO-AFDye 647 spin trap was added in two different ways. In the pre-addition method, CAMPO-AFDye 647 was added in the continuous phase/mayonnaise before oxidation. In the post-addition method, CAMPO-AFDye 647 was added in the continuous phase/mayonnaise after oxidation. For the post addition method samples were imaged at room temperature $\left(21^{\circ} \mathrm{C}\right)$ within one hour after incubation. For the samples used for imaging of protein localisation, $1 \mu \mathrm{L}$ of Rhodamine B stock solution $(10 \mu \mathrm{M})$ was added to $9 \mu \mathrm{L}$ of emulsions to stain proteins before measurements.

Light microscopy. All optical imaging was performed on a home-built microscope, the miCube (Martens et al., 2019), at room temperature $\left(21^{\circ} \mathrm{C}\right)$. For brightfield imaging, we used a LED lamp in transmission mode. For the measurements of CAMPO-AFDye 647 and autofluorescence from oxidised proteins, we used a rescan confocal microscopy (RCM) module (Confocal.nl, Amsterdam, The Netherlands) (De Luca 
et al., 2013) connected to the miCube. Four different lasers were available $(405 \mathrm{~nm}, 488 \mathrm{~nm}, 561 \mathrm{~nm}, 642 \mathrm{~nm}$, Omicron LightHub). The microscope is further equipped with a $100 \mathrm{x}$ oil immersion objective lens (Nikon TIRF 1.49NA HP SR) and an Andor - Zyla 4.2 PLUS sCMOS camera. The frame time was set to $50 \mathrm{~ms}$. We used $2 \mathrm{x} 2$ pixel binning for a final image size of 1024 by 1024 pixels and an effective field of view of $88 \times 88 \mu \mathrm{m}$. Images were recorded using MicroManager 2.0 (Edelstein et al., 2014). To detect protein autofluorescence, samples were excited at $405 \mathrm{~nm}(12 \mathrm{~mW})$. For detection of trapped free radical adducts with CAMPO-AFDye 647, the samples were illuminated of $642 \mathrm{~nm}(34 \mathrm{~mW})$. Fluorescence from Rhodamine B was measured with excitation at $561 \mathrm{~nm}$ $(8 \mathrm{~mW})$. For each measurement, $3 \mu \mathrm{L}$ of the continuous phase sample was added to a well of silicon gasket (CultureWell ${ }^{\mathrm{TM}}$, GRACEBIO-LABS) on a cleaned cover glass.

Sample preparation for cryo-TEM. Samples for cryo-TEM were prepared by applying $3 \mu \mathrm{L}$ of the continuous phase separated from mayonnaise on a 200 mesh $\mathrm{Cu}$ grid with a $\mathrm{R} 2 / 2$ Quantifoil@ carbon support film (Quantifoil MicroTools GmbH). An automated vitrification robot (Thermo Fisher Scientific Vitrobot $^{\mathrm{TM}}$ Mark IV) was used to first blot and then plunge the samples into liquid ethane. Prior to application of the sample the TEM grid was glow-discharged to render the surface of the carbon TEM support film hydrophilic. Cryo-TEM imaging was conducted on the TU/e CryoTitan (Thermo Fisher Scientific) which was operated at $300 \mathrm{kV}$ and is equipped with a Field-Emission Gun, a post-GIF $2 \mathrm{k} \times 2 \mathrm{k}$ Gatan CCD camera, and a post column Gatan Energy Filter (GIF). CryoTEM images were acquired at an electron dose rate of 3 and $6 \mathrm{e}^{-}$ $\AA^{-2} \mathrm{~s}^{-1}$ with an exposure time of $1 \mathrm{~s}$ at magnifications of $6500 \times$ and $24000 \times$, respectively. The nominal defocus was set to $-20 \mu \mathrm{m}$ for $6500 \times$ and $-5 \mu \mathrm{m}$ for $24000 \times$. For detailed imaging conditions for cryo-electron tomography (cryo-ET), see Supplementary Fig. S2.

\section{Results}

Impact of oxidation on multi-scale structure in the continuous phase. We first characterised the aggregation of LDL particles in the isolated continuous phase of mayonnaise using bright field light microscopy over a duration of 5 days. On day 0 , no LDL superstructures were visible, suggesting little to no aggregation of LDLs. In their non-aggregated form, LDLs are too small to be visible using diffraction limited bright field imaging (Figure 1A). After 1 day, micrometre sized LDL superstructures become visible, which then grow to fractals (day 3) and fractal aggregates (day 5). This aggregation behaviour upon oxidation is similar to the one observed for LDLs superstructures from human plasma (Xu and Lin, 2001).

Using cryo-TEM, we determined the diameter of dispersed LDLs to be $36 \pm 6 \mathrm{~nm}$ on day 0 (Figure 1B, 1C, and 1D-I). The number $(N)$ of particles analysed was $N=112$ (see Supplementary Fig. S3). In addition, we saw aggregated LDLs (Figure 1D-II), liposomes characterised by a double phospho- lipid layer without proteins (Figure 1D-III), and spherical, low contrast objects of about $200 \mathrm{~nm}$ in diameter (Figure 1D-IV), which we tentatively assigned to very low-density lipoprotein (VLDL) particles (Huopalahti et al., 2007; Evans et al., 1973).

After one day, we observed aggregates with a broad size distribution composed of mainly LDLs and liposomes. The average diameter of the longest axis was roughly $400 \mathrm{~nm}$ with a standard deviation of $200 \mathrm{~nm}(N=69)$. The structure of these aggregates can be further resolved in cross sections from the acquired electron tomograms (see Supplementary Fig. S2). After 3 and 5 days we observed aggregates spanning the entire $2 \mu \mathrm{m}$ wide hole of the carbon film. Our cryo-TEM data confirm that the superstructures seen in bright field light microscopy consist of LDLs.

Co-localisation of protein oxidation and radical formation in the continuous phase. . LDLs show autofluorescence in the ultraviolet (UV) region mainly due to tryptophan (Trp) residues present in apolipoproteins. Upon oxidation of LDLs, fluorescence emission increases in the visible region due to reactions between lipid peroxidation products such as aldehyde with amino groups (Koller et al., 1986; Schuh et al., 1978). We previously assessed protein oxidation in mayonnaise via autofluorescence using $488 \mathrm{~nm}$ excitation and detection in the 500-560 nm range (Yang et al., 2020). To confirm the decrease of $\mathrm{FL}$ in the $\mathrm{UV}$ region and the increase in longer wavelength, we compared the autofluorescence spectrum after excitation at $300 \mathrm{~nm}, 360 \mathrm{~nm}$, and $405 \mathrm{~nm}$ (see Supplementary Fig. S4). The measurement shows an inverse correlation between the decrease of tryptophane autofluorescence (excitation at $300 \mathrm{~nm}$ ) and the increase of the autofluorescence signal of aldehyde-apolipoprotein adducts (excitation at 405 $\mathrm{nm}$ ). Here, we excited at $405 \mathrm{~nm}$ to maximise the detectable fluorescence emission intensity (see Supplementary Fig. S5). LDL superstructures in the continuous phase showed aggregation over time leading to small fractals upon oxidation with auto-fluorescence (see Supplementary Fig. S6).

To further localise protein oxidation, we deployed adducts of CAMPO, derivative of the spintrap DMPO. CAMPO was either labelled with AFDye 647 for fluorescence imaging or with an $\mathrm{Au}$ nanoparticle for cryo-TEM. We first monitored the accumulation of CAMPO-AFDye 647 fluorescence in the continuous phase of mayonnaise over time. To show the co-localisation between spintraps and oxidised proteins, we combined fluorescence images from CAMPO-AFDye 647 accumulation (red channel) and autofluorescence (blue channel). Two different ways of adding CAMPO-AFDye 647 to the continuous phase were used to see the effect of accumulation of CAMPO during incubation: pre-addition (Figure 2A) and post-addition (Figure 2B).

On day 0 of the pre-addition series, we did not observe any accumulation of intensities caused by either protein autofluorescence or CAMPO-AFDye 647 fluorescence (see Supplementary Fig. S7). The high intensities in the red channel indicate that CAMPO-AFDye 647 is freely diffusing in the continuous phase 


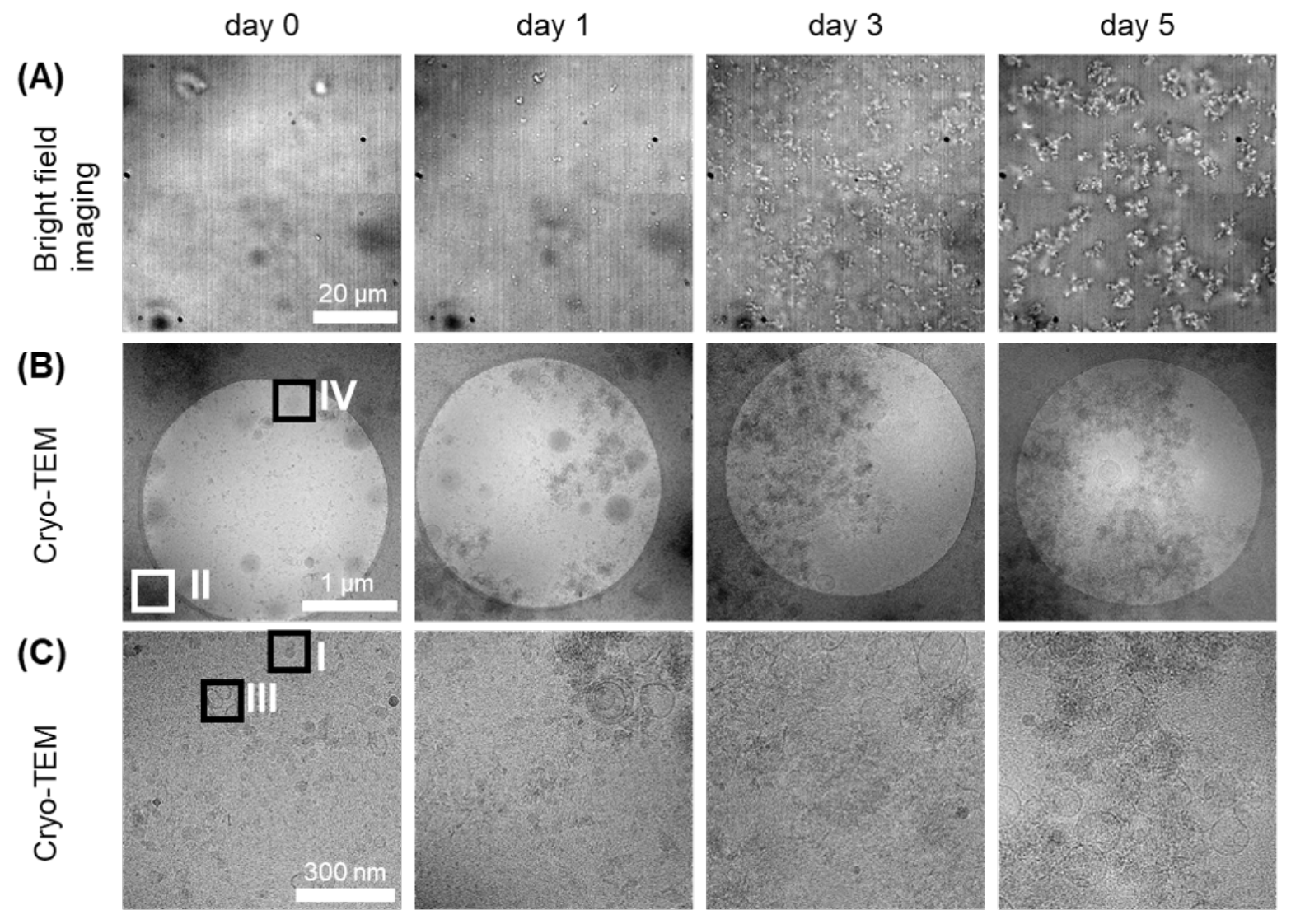

(D) Cryo-TEM - day 0

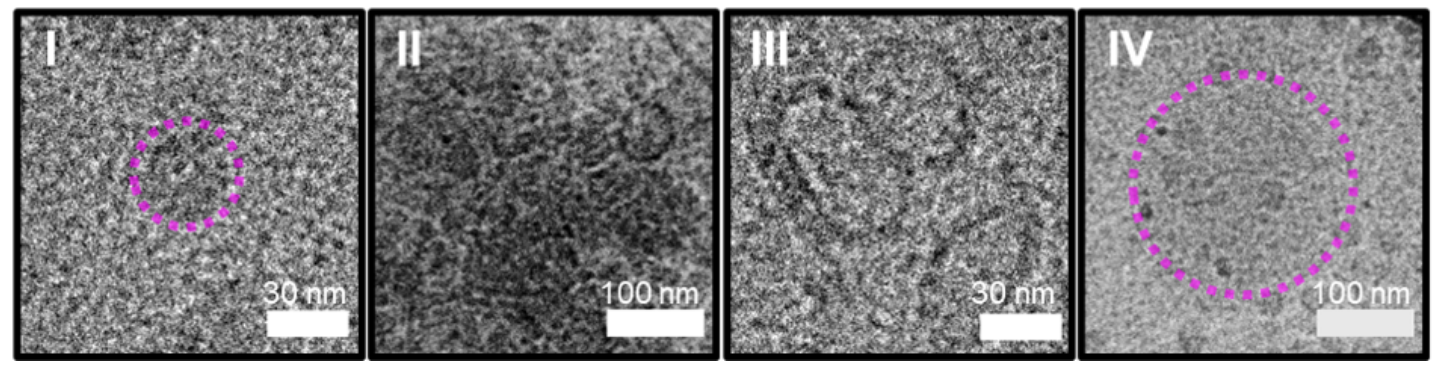

Fig. 1. Multi-scale imaging of LDL particles aggregation in the continuous phase of mayonnaise. (A) Bright field images after incubation for 0, $1,3,5$ days at $40^{\circ} \mathrm{C}$. Black blurry spots located in the same place of different images indicate dust on the camera, not from the sample itself. Cryo-TEM images of the continuous phase at magnifications of $6500 \times(\mathbf{B})$ and $24000 \times(\mathbf{C})$ during the same sample incubation period. (D) Zoom-in regions from (B) and (C) for day 0 showing I: LDL, II: aggregated LDLs, III: liposome vesicles, IV: spherical low-contrast object, VLDLs. A nominal defocus of $-20 \mu \mathrm{m}$ and $-5 \mu \mathrm{m}$ was applied for magnifications of $6500 \times$ and $24000 \times$, respectively. Contrast and brightness were adjusted for visibility. Some images (D I and D IV) contain dashed outlines (magenta) of the underlying structures for visual guidance.

instead of reacting with protein free radicals. Similar to the bright field images seen in Figure 1A, LDL superstructures and aggregates form over time (Figure 2A). Further, the autofluorescence (blue) increases and CAMPO-AFDye 647 (red) accumulates in the LDL aggregates. The decrease of the background in the continuous phase is in line with CAMPO-AFDye 647 accumulating at sites with protein radicals. After 5 days, CAMPO-AFDye 647 accumulation in fractal aggregates of LDL superstructures was observed and found to be mostly co-localised with autofluorescence from oxidised proteins.

In the post-addition series (Figure 2B), we could measure increased autofluorescence (blue), but noticed elevated back- ground intensities originating from freely diffusing CAMPOAFDye 647 . Over the 5 days, aggregates in the continuous phase from both addition methods showed a similar extent of aggregation. Figure $2 \mathrm{C}$ compares spectrally resolved channels for samples oxidised for 5 days. Fluorescence of accumulated CAMPO-AFDye 647 and protein autofluorescence are clearly co-localised in the pre-addition sample whereas the signal to noise ratio of CAMPO-AFDye 647 is compromised in the postaddition sample due to the fluorophore still being present in free solution. We note that autofluorescence signals from two different addition methods showed similar intensities indicating they are not influenced by CAMPO-addition. 


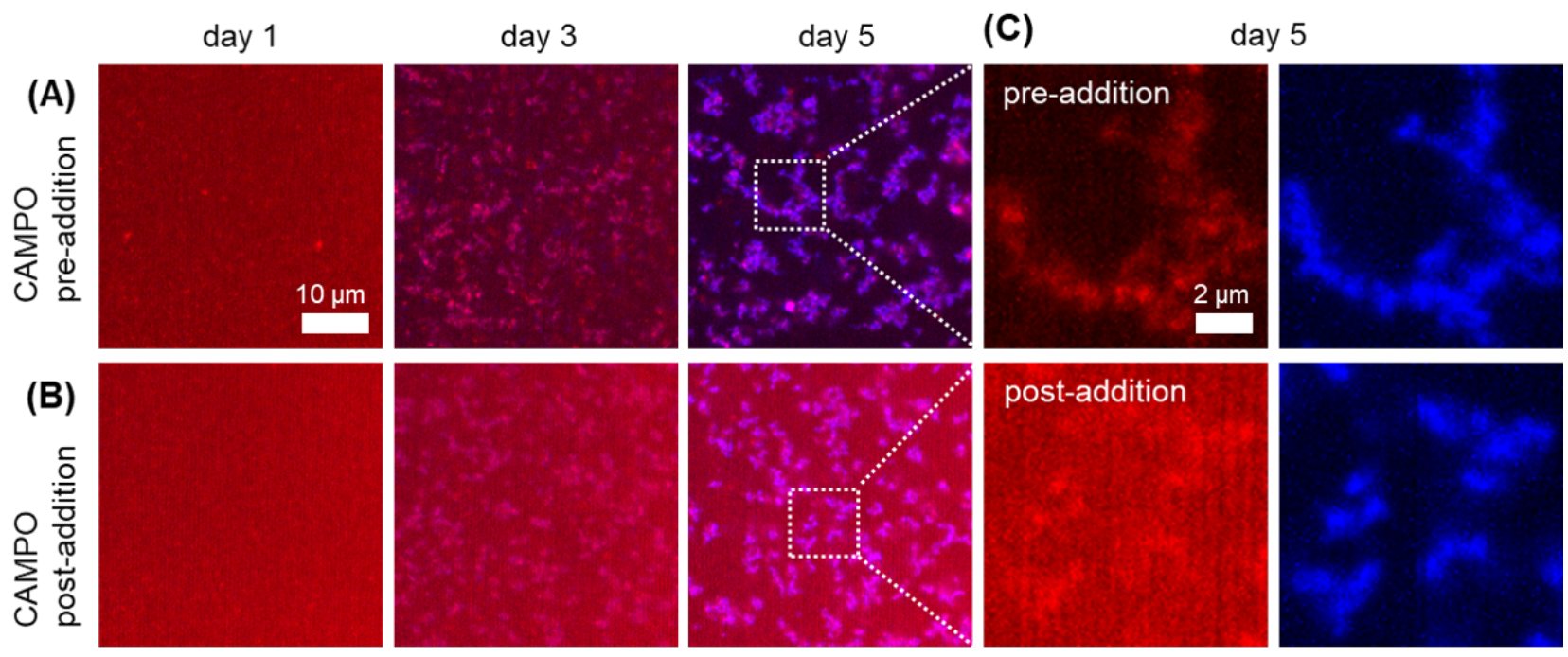

Fig. 2. Fluorescence microscopy images of continuous phases separated from mayonnaise. Images recorded zero and after one, three, and five days are shown with the red (CAMPO-AFDye 647) and blue (autofluorescence) channels combined from re-scan confocal measurements. Intensities are normalized in the same channel. (A, B) CAMPO-AFDye 647 was pre-added before oxidation (A) and post-added after continuing oxidation (B). No oxidation is observable at day 0. CAMPO-AFDye 647 accumulates in the continuous phase of mayonnaise and and aggregates of LDLs show the co-localisation of CAMPO-AFDye 647 (red) and autofluorescence (blue). (C) Zoom-in (white dashed box) from (A) and (B). Spectrally resolved channels from samples after 5 days of oxidation.

We further investigated whether the CAMPO-Au nanoparticles (CAMPO-AuNPs) co-localise with aggregated LDLs. Cryo-TEM images indicate that CAMPO-AuNPs favour the proximity of aggregated proteins, while the control experiments with non-functionalised AuNPs did not show such behaviour (see Supplementary Fig. S8). We note that the low stock concentration of the CAMPO-AuNPs dispersion $\left(\mathrm{AU}_{520} \approx\right.$ 0.2 ) prevented us from further quantifying the localisation of CAMPO-AuNPs close to aggregated proteins.

\section{Co-localisation of protein oxidation and radical formation in} dilute mayonnaise. The dense packing of oil droplets made it difficult to discern LDLs in the continuous phase and at the interfaces of oil droplets. The samples were therefore diluted before observing protein autofluorescence and CAMPOAFDye 647 accumulation. As a control, dilute $(26 \%(\mathrm{w} / \mathrm{w})$ oil concentration) mayonnaise without CAMPO-AFDye 647 was measured (Figure 3A). As seen, the level of autofluorescence at the oil/water droplet interface and in the continuous phase increased upon oxidation from day 0 to day 5 . Images of the red channel (CAMPO-AFDye 647) and the blue channel (autofluorescence) show co-localisation of accumulated spintraps and oxidized proteins. Already on day 0, we observed accumulation of CAMPO-AFDye 647 fluorescence at the oil/water droplet interface and in the continuous phase between droplets (Figure 3B). On day 5, we saw an increase in protein oxidation around and especially between droplets, likely representing LDL aggregates (Figure 3B). It should be noted that although in most cases co-localisation of CAMPOAFDye 647 and auto-fluorescence was observed, few regions showed only CAMPO-AFDye 647 accumulation. In Figure 3 C$\mathrm{D}$ selected regions of interest and spectrally separated images are shown. On day 0, only CAMPO-AFDye 647 accumulated heterogeneously at the oil/water droplet interface whereas on day 5 , the autofluorescence signal around the droplets partially co-localise with CAMPO-AFDye 647 (Figure 3C and 3D). The accumulation of CAMPO-AFDye 647 at the droplet interface as seen in Figure 3D occurs in micronscale domains as well as a thin homogeneous halo around the droplet interface. These findings suggest that the accumulation of CAMPO-AFDye 647 spintraps occurs in both micron-scale LDL granules and protein molecules stabilizing the droplet interfaces as emulsifiers. Both apoproteins originating from LDL particles and phosvitin qualify for such a role.

To validate that the accumulation of CAMPO-AFDye 647 at droplet interfaces is due to protein free radical formation induced by nearby lipid radicals, the $\mathrm{pH}$ of the mayonnaise was varied. Under the usual acidic environment of mayonnaise, phosvitin has weak affinity for ferric ions which allows them to act as a catalysts for lipid oxidation at oil/water droplet interfaces (Merkx et al., 2019). At neutral pH, phosvitin has a stronger binding affinity for ferric ions than acidic $\mathrm{pH}$ thus preventing ferric ions to be released at the interface (Castellani et al., 2004). Indeed, whereas accumulation of CAMPO-AFDye 647 at droplet interfaces at $\mathrm{pH} 4.0$ (Figure 4A) was seen, we did not observe this at pH 6.6 (Figure 4B). Furthermore, Rhodamine B staining confirmed that proteins are present at the droplet interfaces both at $\mathrm{pH} 4.0$ and 6.6 (Figure 4C and 4D). This observation ruled out potential effects due to $\mathrm{pH}$ induced release of proteins from the droplet interface. Co-localisation of Rhodamine B and CAMPO-AFDye 647 could only be observed in acidic mayonnaise (Figure $4 \mathrm{E}$ and $4 \mathrm{~F})$.

The accumulation of CAMPO-AFDye 647 on day 0 (Figure 


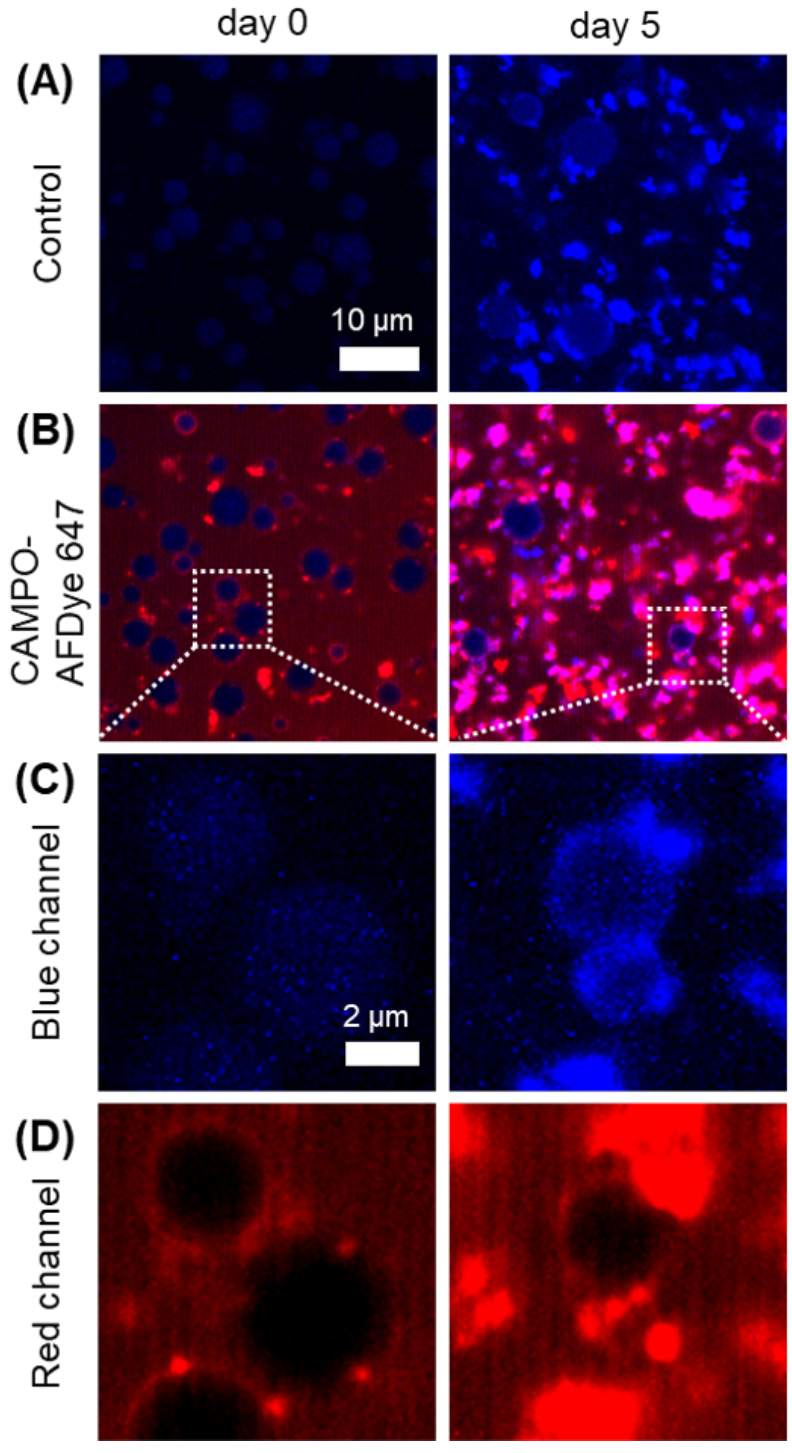

Fig. 3. Localisation of CAMPO-AFDye 647 and autofluorescence in dilute mayonnaise. Images combined from red (CAMPO-AFDye 647) and blue (autofluorescence) emission channels are shown in (A)-(B). Stripped soybean oil was used. (A) Dilute emulsions without any fluorescent dyes measured on day 0 and day 5 after incubation. These control samples only showed autofluorescence after incubation. (B) Samples with the post-addition of $1 \mu \mathrm{M}$ CAMPO-AFDye 647. CAMPO-AFDye 647 was added just before each measurement. Co-localisation between CAMPOAFDye 647 and autofluorescence were seen after 5 days incubation. (C) Zoom-in (white dashed box) blue channel images from (B). Significant increased auto-fluorescence were observed only in the sample after 5 days oxidation. (D) Zoom-in (white dashed box) red channel images from (B). Accumulation of CAMPO-AFDye 647 at the interface was measured after 5 days oxidation whereas small granules of CAMPOAFDye 647 were observed in the fresh sample. Scales of fluorescence intensities are adjusted in the same range for each channel to see the increased CAMPO-AFDye 647 accumulation and autoFL.
3B) indicated that protein radicals are already formed soon (1 hour) after preparing the dilute mayonnaise. In order to exclude that this effect is induced by aspecific binding of CAMPO-AFDye 647, we prepared dilute mayonnaise with and without EDTA, which acts as a strong antioxidant by chelating metal ions from phosvitin at the droplet interface

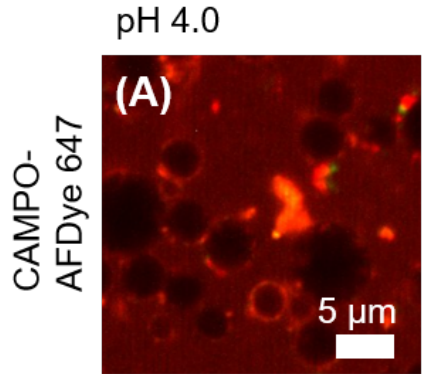

$\mathrm{pH} 6.6$
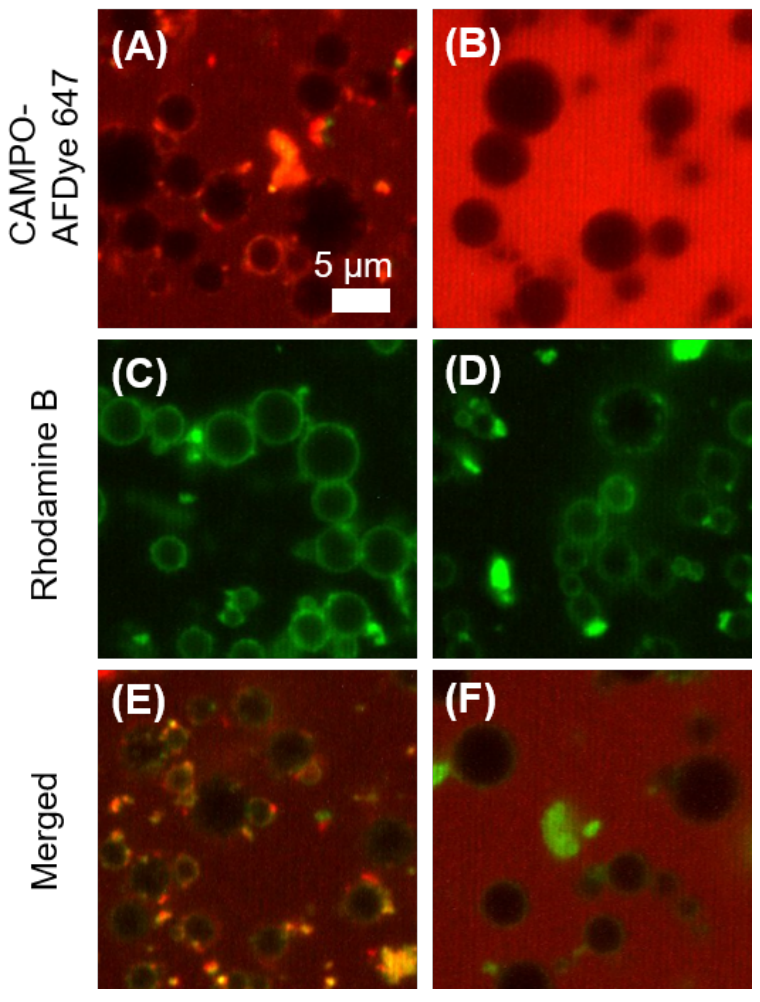

Fig. 4. $\mathrm{pH}$ dependency of CAMPO-AFDye 647 accumulation with stained proteins in dilute mayonnaise. $1 \mu \mathrm{M}$ of CAMPO-AFDye 647 and Rhodamine $\mathrm{B}$ were added before measurements in samples stored at $4^{\circ} \mathrm{C}$. Corresponding bright field images can be found in Supplementary Fig. S9. (A, B) Accumulation of CAMPO-AFDye 647 with excitation at 640 nm. (C, D) Protein staining with Rhodamine B at the oil/water droplet interface and in the continuous phase. Samples were illuminated at 561 nm. (E, F) Overlay images of CAMPO-AFDye 647 and Rhodamine B. Co-localisation is observed in dilute mayonnaise only at $\mathrm{pH} 4.0$.

(Merkx et al., 2019). Upon addition of EDTA, less CAMPOAFDye 647 accumulated at the droplet interfaces over time compared to conditions in absence of EDTA (Figure 5A and 5B). Moreover, the dilute mayonnaise without EDTA showed an increase in the number and the size of the LDL aggregates over time (Figure 5A), while in the emulsion containing EDTA only an increase in the number of the aggregates was detected (Figure 5B). A zoom into the images after 10 days of oxidation showed that in absence of EDTA most of droplet interfaces feature trapped CAMPO-AFDye 647 as they show halos at the interface (Figure 5C). In the presence of EDTA, accumulation at interfaces is virtually absent. Accumulation in aggregates is sill visible in LDL aggregates, but less pronounced than in absence of EDTA. This observation confirmed that the addition of EDTA significantly delayed lipid radical formation and subsequent protein oxidation at the interface but was less effective to inhibit oxidation of LDLs in the continuous phase.

\section{Discussion}

Our multi-scale microscopy measurements suggest an pathway for protein oxidation including LDLs in the continuous phase 


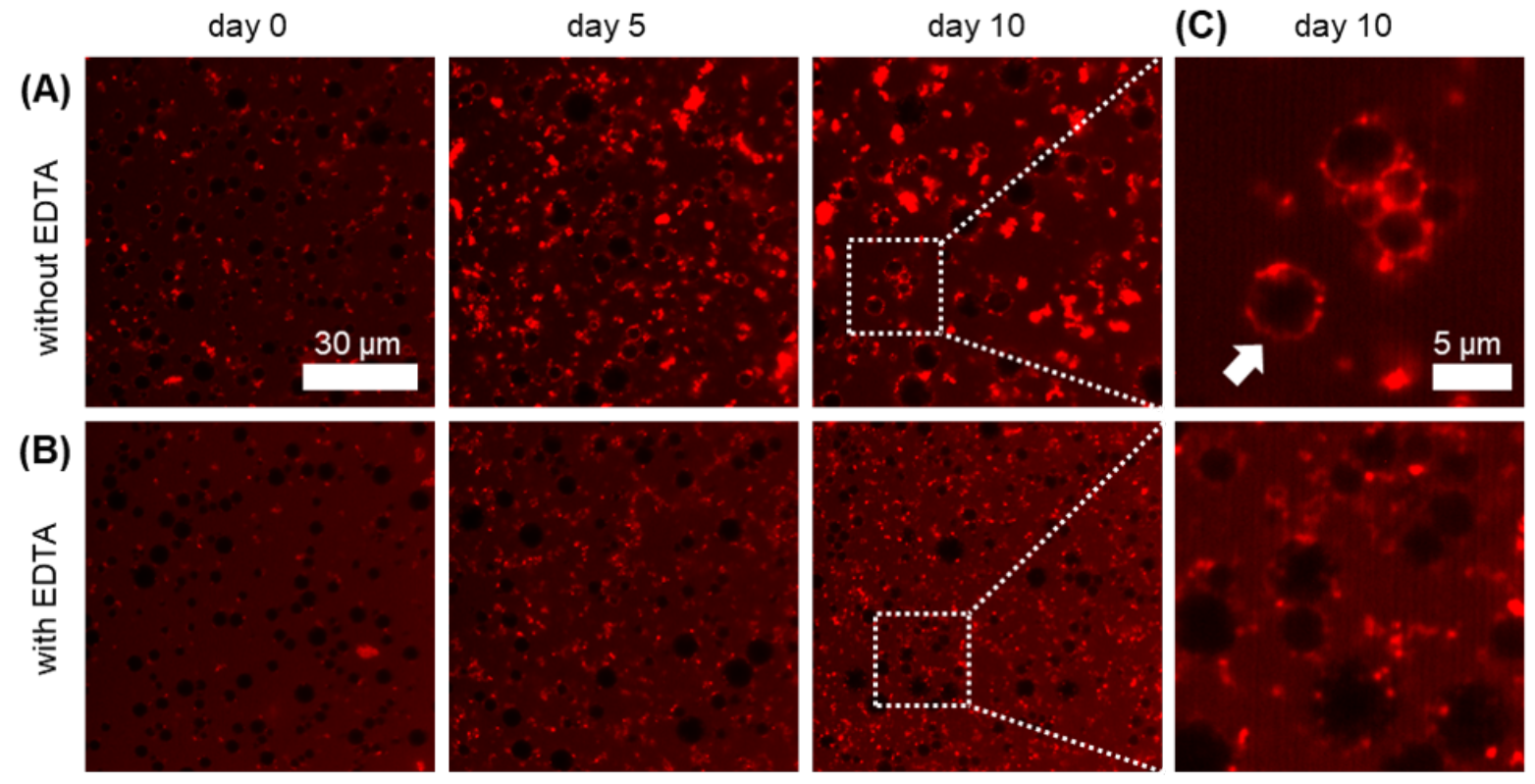

Fig. 5. EDTA dependency of CAMPO-AFDye 647 accumulation in dilute mayonnaise at pH 4.0. The CAMPO-AFDye 647 spintrap was added at the indicated time points (post-addition). Images were measured using RCM. (A, B) Dilute mayonnaise without (A) and with 100 mM EDTA (B) measured after 0, 5 and 10 days of incubation. (C) Zoom-in (white dashed box) images from (A, B) after 10 days oxidation. The arrow points the halos at the oil/water droplet interface from CAMPO-AFDye 647 accumulation.

of mayonnaise which is indepedent from lipid oxidation in oil droplets. Free radical-mediated oxidation of unsaturated fatty acids in LDL leads to a chain reaction and triggers protein oxidation and aggregation (Figure 1). Increased auto fluorescence intensity supports this finding (Supplementary Fig. S4) as it was previously shown that increased autoFL from oxidised LDL is due to the reaction between lipid peroxidation products such as aldehyde with amino groups (Koller et al., 1986; Schuh et al., 1978). An increase in autofluorescence alone is, however, not a suitable marker for the early stage of oxidation (Figure 2) as aldehydes are secondary breakdown products of unsaturated lipids. Therefore, we here decided to use a water-soluble fluorescent spin trap (CAMPO-AFDye 647 ) that specifically targets protein radicals. We assume that due to the immobility of proteins at the oil/water droplet interfaces or in LDL particles, the trapped radicals accumulate locally. This mechanism is unlikely to work for spin trapped lipid radicals which are smaller and more likely to diffuse throughout oil droplets. Our results suggest, however, that spin trapping of protein radicals can be used as a proxy for local and early lipid radical formation at droplet interfaces (Figure 3) and in LDL particles in the continuous phase (Figure 2 ). Whereas protein autofluorescence showed up in the late stage of oxidation, the spintrap was already visible in fresh emulsions (Figure 3).

The data in the Supplementary Fig. S7 did not yet show accumulation of CAMPO-AFDye 647 in the continuous phase on day 0 . This implies that protein oxidation at the oil/water droplet interfaces is faster due to the generation of lipid radicals within the droplets. Figure 6 summarises our findings in a schematic manner. We discern two spatially separate mechanisms by which protein radicals can be formed by lipid radicals. At the oil/water droplet interfaces, lipid oxidation is catalysed by iron ions associated with surface bound phosvitin (Ghorbani Gorji et al., 2016; Causeret et al., 1991). The catalytic activity of iron close to the interface is increased at low $\mathrm{pH}$ of mayonnaise, as the binding strength of ions to phosvitin is decreased (Figure 6A) (Thomson et al., 2000; Jacobsen et al., 1999; Takeuchi et al., 2022). Iron ions can also be outcompeted from phosvitin by chelation with EDTA (Thomson et al., 2000). In a spatially separate mechanism, lipid oxidation in LDL particles produces lipid radicals that induce protein free radical formation and protein oxidation (Figure 6B). This mechanism is still active in the presence of EDTA, suggesting that chelated iron can still act as catalyst albeit less strong as in their free form. Upon oxidation, LDL particles are prone to aggregation (Figure 6C) (Witztum and Steinberg, 1991; Xu and Lin, 2001), which can play a role in the physical instability of mayonnaise in prolonged stages of oxidation. To this end, it remains unclear why most but not all areas show co-localisation of CAMPO accumulation with autoFL. One explanation is trapping of CAMPO-AFDye 647 by phosvitin, which contains less tryptophan residues than apoproteins (Byrne et al., 1984; Olofsson et al., 1987). To explore this further, we suggest to employ a higher resolution imaging method (Hohlbein, 2021) in combination with localisation of phosvitin using a phosvitin-antibody conjugated with fluorophores (Jabermoradi et al., 2022). 


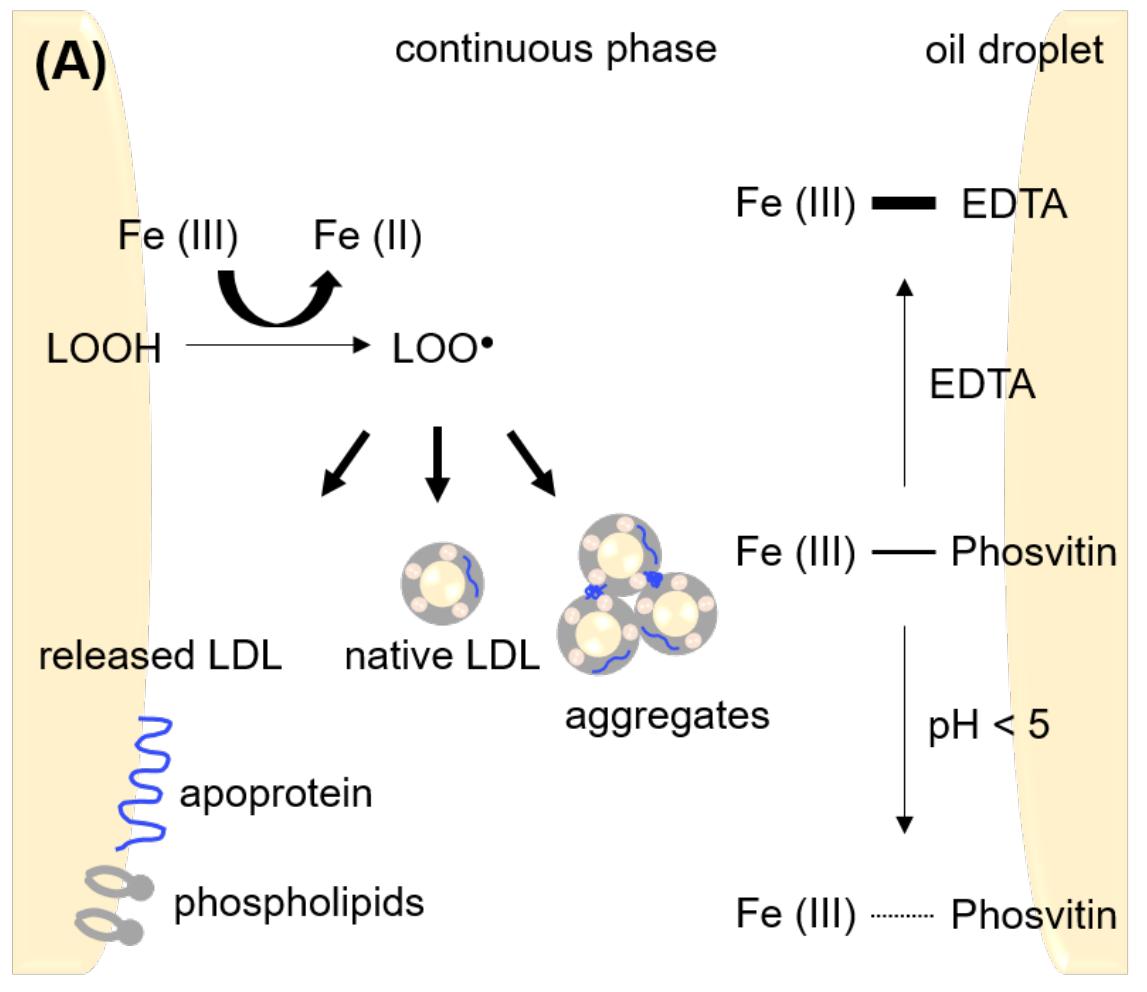

(B) LDL

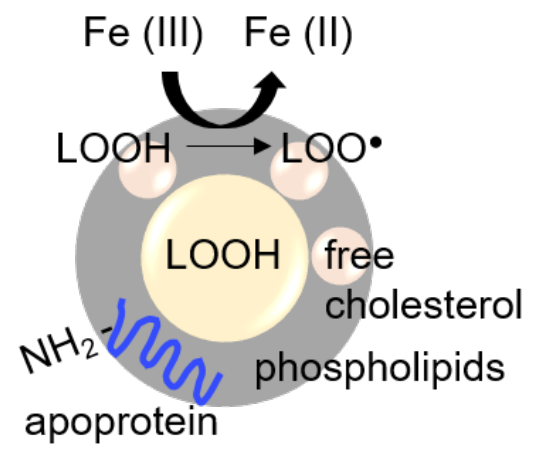

\section{(C) aggregates}

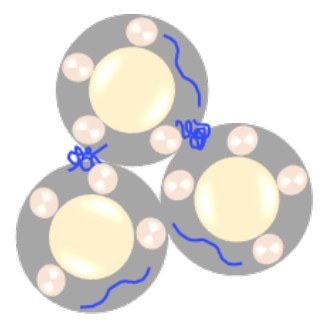

Fig. 6. Schematic of lipid and protein oxidation with formation of free radicals at the oil/water droplet interface and in the continuous phase. Apoproteins and phospholipids relased from LDLs are adsorbed at the interface. Native and oxidised LDLs are present in the continuous phase. (A) Depiction of underlying lipid oxidation mechanisms including oxidation of proteins dispersed at oil/water interface or present in LDLs in different condition (acidic/neutral condition and presence of EDTA). Addition of EDTA delays lipid oxidation because of stronger binding with Fe (III) than phosvitin. In acidic condition, Fe (III) promotes lipid oxidation due to weak binding between Fe (III) and phosvitin. Thick and dashed lines respectively indicate strong and weaker binding of iron with EDTA and phosvitin. (B) Apoproteins in LDL can be oxidised from lipid free radicals generated within the particles and from oil droplets at the interface. (C) Oxidised LDL can cause protein changes such as misfolding and/or cross linking and aggregation of LDLs at the interface and in the continuous phase.

\section{Conclusion}

We demonstrated that the accumulation of a fluorescently labelled spintrap (CAMPO-AFDye 647) can be used to localise protein radical formation at droplet interfaces and in the continuous phase of mayonnaise. The local formation of protein radicals can be detected ahead of an increase in autofluorescence and can be used as a proxy for local formation of lipid radicals.

In mayonnaise, protein free radicals can be generated via two routes: at the droplet interface, oxidation of proteins including LDLs, apoproteins, and phosvitin is induced by lipid radicals formed in the droplets. This route is not effective in a high pH environment, at which iron is strongly bound to phosvitin. In the continuous phase, lipids in LDL particles are also prone to generate radicals, inducing protein oxidation and LDL aggregation. Upon addition of EDTA, protein radical formation is more effectively inhibited at the droplet interface as in the continuous phase.

\section{Authors' Contributions}

S.Y.: Investigation, Validation, Visualization, Writing - original draft. M.T.: Investigation, Validation, Visualization, Writing - original draft. H.F.: Conceptualization, Supervision,
Writing - review \& editing. J.P.M.v.D: Conceptualization, Supervision, Writing - review \& editing. J.H.: Conceptualization, Supervision, Writing - review \& editing.

\section{Competing Interests}

J.P.M.v.D. is employed by a company that manufactures and markets mayonnaise. The other authors declare that they have no known competing financial interests or personal relationships that could influence the work reported in this paper.

\section{Funding Statement}

This work was financially supported by the Netherlands Organisation for Scientific Research (NWO) in the framework of the Innovation Fund for Chemistry and from the Ministry of Economic Affairs in the framework of the "TKI/PPSToeslagregeling", with grant number of 731.017.301.

\section{Data availability}

The experimental raw data is currently available upon request and will be made available on https://zenodo.org. 


\section{Acknowledgements}

We would like to thank our colleagues in the LICENSE consortium for helpful discussions.

\section{References}

Anton, M. (2013). Egg yolk: Structures, functionalities and processes. Journal of the Science of Food and Agriculture, 93(12):2871-2880.

Anton, M., Martinet, V., Dalgalarrondo, M., Beaumal, V., David-Briand, E., and Rabesona, H. (2003). Chemical and structural characterisation of low-density lipoproteins purified from hen egg yolk. Food Chemistry, 83(2):175-183.

Berton, C., Genot, C., and Ropers, M. H. (2011). Quantification of unadsorbed protein and surfactant emulsifiers in oil-in-water emulsions. Journal of Colloid and Interface Science, 354(2):739-748.

Berton, C., Ropers, M. H., Guibert, D., Solé, V., and Genot, C. (2012). Modifications of interfacial proteins in oil-in-water emulsions prior to and during lipid oxidation. Journal of Agricultural and Food Chemistry, 60(35):8659-8671.

Berton-Carabin, C. C., Ropers, M. H., and Genot, C. (2014). Lipid Oxidation in Oil-in-Water Emulsions: Involvement of the Interfacial Layer. Comprehensive Reviews in Food Science and Food Safety, 13(5):945-977.

Byrne, B. M., van het Schip, A. D., Van deKlundert, J. A., Arnberg, A. C., Gruber, M., and AB, G. (1984). Amino Acid Sequence of Phosvitin Derived from the Nucleotide Sequence of Part of the Chicken Vitellogenin Gene. Biochemistry, 23(19):4275-4279.

Castellani, O., Guérin-Dubiard, C., David-Briand, E., and Anton, M. (2004). Influence of physicochemical conditions and technological treatments on the iron binding capacity of egg yolk phosvitin. Food Chemistry, 85(4):569-577.

Causeret, D., Matringe, E., and Lorient, D. (1991). Ionic Strength and pH Effects on Composition and Microstructure of Yolk Granules. Journal of Food Science, 56(6):1532-1536.

De Luca, G. M., Breedijk, R. M., Brandt, R. A., Zeelenberg, C. H., de Jong, B. E., Timmermans, W., Azar, L. N., Hoebe, R. A., Stallinga, S., and Manders, E. M. (2013). Re-scan confocal microscopy: scanning twice for better resolution. Biomedical Optics Express, 4(11):2644-2656.

Edelstein, A. D., Tsuchida, M. A., Amodaj, N., Pinkard, H., Vale, R. D., and Stuurman, N. (2014). Advanced methods of microscope control using $\mu$ Manager software. Journal of Biological Methods, 1(2):1-18.

Evans, R. J., Bauer, D. H., Bandemer, S. L., Vaghefi, S. B., and Flegal, C. J. (1973). Structure of egg yolk very low density lipoprotein. Polydispersity of the very low density lipoprotein and the role of lipovitellenin in the structure. Archives of Biochemistry and Biophysics, 154(2):493-500.

Ghorbani Gorji, S., Smyth, H. E., Sharma, M., and Fitzgerald, M. (2016). Lipid oxidation in mayonnaise and the role of natural antioxidants: A review. Trends in Food Science and Technology, 56:88-102.

Hohlbein, J. (2021). Single-molecule localization microscopy as an emerging tool to probe multiscale food structures. Food Structure, 30:100236.

Huopalahti, R., López-Fandiño, R., Anton, M., and Schade, R. (2007). Bioactive Egg Compounds. Springer-Verlag Berlin Heidelberg, 1 edition. Jabermoradi, A., Yang, S., Gobes, M. I., van Duynhoven, J. P., and Hohlbein, J. (2022). Enabling single-molecule localization microscopy in turbid food emulsions. Philosophical Transactions of the Royal Society A, 380(2220):20200164.

Jacobsen, C., Adler-Nissen, J., and Meyer, A. S. (1999). Effect of ascorbic acid on iron release from the emulsifier interface and on the oxidative flavor deterioration in fish oil enriched mayonnaise. Journal of Agricultural and Food Chemistry, 47(12):4917-4926.

Koller, E., Quehenberger, O., Jürgens, G., Wolfbeis, O. S., and Esterbauer, $H$. (1986). Investigation of human plasma low density lipoprotein by threedimensional flourescence spectroscopy. FEBS Letters, 198(2):229-234.

Lee, J. and Decker, E. A. (2011). Effects of metal chelator, sodium azide, and superoxide dismutase on the oxidative stability in riboflavinphotosensitized oil-in-water emulsion systems. Journal of Agricultural and Food Chemistry, 59(11):6271-6276.

Martens, K. J., van Beljouw, S. P., van der Els, S., Vink, J. N., Baas, S., Vogelaar, G. A., Brouns, S. J., van Baarlen, P., Kleerebezem, M., and Hohlbein, J. (2019). Visualisation of dCas9 target search in vivo using an open-microscopy framework. Nature Communications, 10(1):1-11.

Mason, R. P. (2016). Imaging free radicals in organelles, cells, tissue, and in vivo with immuno-spin trapping. Redox Biology, 8:422-429.

Mason, R. P. and Ganini, D. (2019). Immuno-spin trapping of macro- molecules free radicals in vitro and in vivo - One stop shopping for free radical detection. Free Radical Biology and Medicine, 131:318-331.

Merkx, D. W., Delić, F., Wierenga, P. A., Hennebelle, M., and van Duyn hoven, J. P. (2019). 31P NMR assessment of the phosvitin-iron complex in mayonnaise. Magnetic Resonance in Chemistry, 57(9):540-547.

Mizutani, R. and Nakamura, R. (1985). Physical State of the Dispersed Phases of Emulsions Prepared with Egg Yolk Low Density Lipoprotein and Bovine Serum Albumin. Journal of Food Science, 50(6):1621-1623.

Nielsen, N. S., Petersen, A., Meyer, A. S., Timm-Heinrich, M., and Jacobsen, C. (2004). Effects of lactoferrin, phytic acid, and EDTA on oxidation in two food emulsions enriched with long-chain polyunsaturated fatty acids. Journal of Agricultural and Food Chemistry, 52(25):7690-7699.

Olofsson, S. O., Bjursell, G., Boström, K., Carlsson, P., Elovson, J., Prot ter, A. A., Reuben, M. A., and Bondjers, G. (1987). Apolipoprotein B: structure, biosynthesis and role in the lipoprotein assembly process. Atherosclerosis, 68(1-2):1-17.

Pinchuk, I. and Lichtenberg, D. (1999). Copper-induced LDL peroxidation: Interrelated dependencies of the kinetics on the concentrations of copper, hydroperoxides and tocopherol. FEBS Letters, 450(3):186-190.

Pinchuk, I. and Lichtenberg, D. (2002). The mechanism of action of antioxidants against lipoprotein peroxidation, evaluation based on kinetic experiments. Progress in Lipid Research, 41(4):279-314.

Schaich, K. (2008). Co-oxidations of oxidizing lipids: Reactions with proteins. In Kamal-Eldin, A. and Min, D., editor, Lipid Oxidation Pathways, volume 2, pages 183-274. AOCS Press.

Schaich, K. M. (2012). Thinking outside the classical chain reaction box of lipid oxidation. Lipid Technology, 24(3):55-58.

Schaich, K. M. and Karel, M. (1976). Free radical reactions of peroxidizing lipids with amino acids and proteins: An ESR study. Lipids, 11(5):392400

Schuh, J., Fairclough, G. F., and Haschemeyer, R. H. (1978). Oxygen mediated heterogeneity of apo-low-density lipoprotein. Proceedings of the National Academy of Sciences of the United States of America 75(7):3173-3177.

Sirvente, H., Beaumal, V., Gaillard, C., Bialek, L., Hamm, D., and Anton, M. (2007). Structuring and Functionalization of Dispersions Containing Egg Yolk, Plasma and Granules Induced by Mechanical Treatments. Journal of Agricultural and Food Chemistry, 55(23):9537-9544.

Takeuchi, M., Mashima, T., Sztucki, M., Petukhov, A. V., Vis, M., Friedrich, H., and Tuinier, R. (2022). Characterization of hen phosvitin in aqueous salt solutions: Size, structure, and aggregation. Food Hydrocolloids, page 107545.

Thomson, M. K., Jacobsen, C., and Skibsted, L. H. (2000). Mechanism of initiation of oxidation in mayonnaise enriched with fish oil as studied by electron spin resonance spectroscopy. European Food Research and Technology, 211(6):381-386.

Towner, R. A., Smith, N., Saunders, D., Henderson, M., Downum, K., Lupu, F., Silasi-Mansat, R., Ramirez, D. C., Gomez-Mejiba, S. E., Bonini, M. G. Ehrenshaft, M., and Mason, R. P. (2012). In vivo imaging of immunospin trapped radicals with molecular magnetic resonance imaging in a diabetic mouse model. Diabetes, 61(10):2405-2413.

Wang, Y., Liu, Y., Ma, L., Yang, L., Cong, P., Lan, H., Xue, C., and Xu, J. (2020). Co-oxidation of Antarctic krill oil with whey protein and myofibrillar protein in oil-in-water emulsions. Journal of Food Science, 85(11):37973805

Witztum, J. L. and Steinberg, D. (1991). Role of oxidized low density lipopro tein in atherogenesis. Journal of Clinical Investigation, 88(6):1785-1792.

$\mathrm{Xu}, \mathrm{S}$. and Lin, B. (2001). The mechanism of oxidation-induced lowdensity lipoprotein aggregation: An analogy to colloidal aggregation and beyond? Biophysical Journal, 81(4):2403-2413.

Yang, S., Verhoeff, A. A., Merkx, D. W., van Duynhoven, J. P., and Hohlbein J. (2020). Quantitative spatiotemporal mapping of lipid and protein oxidation in mayonnaise. Antioxidants, 9(12):1-13.

Zhu, Z., Zhao, C., Yi, J., Liu, N., Cao, Y., Decker, E. A., and McClements D. J. (2018). Impact of Interfacial Composition on Lipid and Protein Co-Oxidation in Oil-in-Water Emulsions Containing Mixed Emulisifers Journal of Agricultural and Food Chemistry, 66(17):4458-4468. 